\title{
A New Component Exploration Research Based on Mathematic Model
}

\author{
PENG Lijuan ${ }^{1, a}$ \\ ${ }^{1}$ Chongqing College of Electronic Engineering, Chongqing 401331 \\ a.285459707@qq.com
}

Key words: inductance, capacitor, resistor, gyrator.

\begin{abstract}
With the rapid development of modern scientific technology, the electronic majors such as technology, communication, navigation and automatic control, have great breakthrough and innovation in depth and width. Some new type tip subject need miniaturization of inductance elements and simplification of the memory . This article has the study on the problems mentioned above from the aspect of classical field.
\end{abstract}

\section{Introduction}

With the modern science theory, it is proved that the capacitor incurvate can turn into inductive components. And to realize capacitor super miniaturization, modern science and technology can completely solve it. Therefore the inductive components of miniaturization can be easily solved. As shown in figure 1 of two-port networks, in the 2-2 if a capacitor C/termination, as shown, reference direction.

$$
\dot{i}_{2}=-c \frac{d u_{2}}{d t}
$$

According to the definition of incurvate

$$
\boldsymbol{u}_{1}=-r \boldsymbol{i}_{2}
$$

With the formula 1 and formula 2, we can get:

$$
\begin{aligned}
& u_{1}=r c \frac{d u_{2}}{d t} \text { and } u_{2}=r \boldsymbol{i}_{1} \\
& \text { so } u_{1}=r^{2} c \frac{d i_{1}}{d r}
\end{aligned}
$$

Formula 3 reflects two-port network from 1 1 / depends on the voltage and current fashion shown in figure 1, equivalent inductance coil voltage and current relationship, the equivalent inductance $\mathrm{L}$ is:

$$
\mathrm{L}=r^{2} c
$$

So how to achieve incurvate? Usually there is a lot of the realization methods of rotary. Below two op-amp and some resistance components of circuit are used, as shown in figure 2.

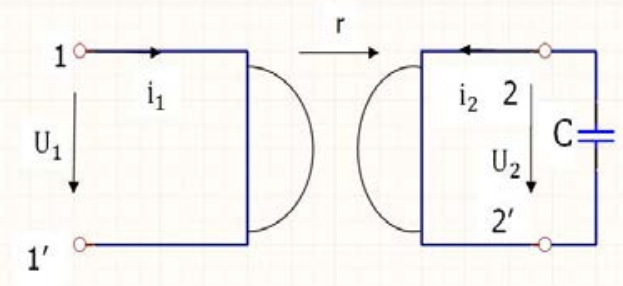

Figure 1: Two-port Network 


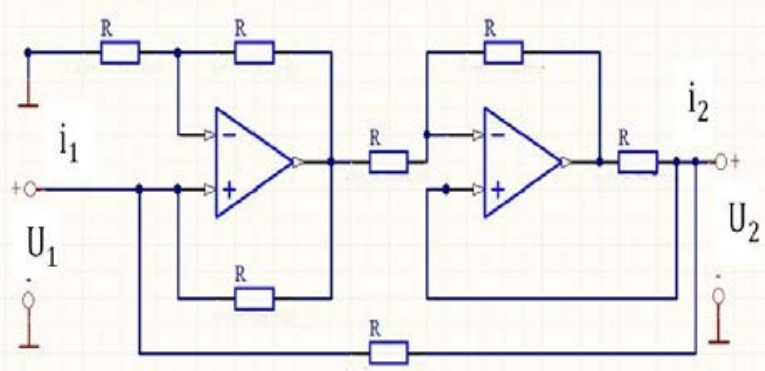

Figure 2: Two-stage Operational Amplifier

This circuit can be regarded as two twoport network of parallel, see figure 3 shows.

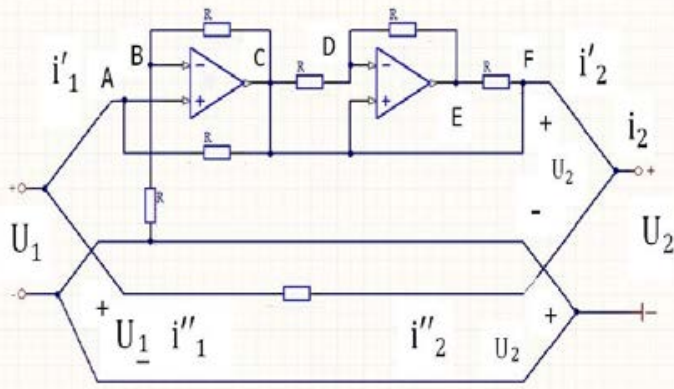

Figure 3: Parallel between Two-port Network and Two-stage Operational Amplifier Presently with the phasor analysis, two of the above circuit amplifier with "O" is referenced, shown in figure 3 ,

$\dot{U}_{A}=\dot{U}_{B}=\dot{U}_{1} \quad \dot{U}_{C}=2 \dot{U}_{1}$

because $\dot{I}_{1}=\frac{\dot{U}_{A}{ }^{-} \dot{U}_{C}}{R}=\frac{\dot{U}_{1}{ }^{-2} \dot{U}_{1}}{R}=\frac{{ }^{-} \dot{U}_{1}}{R}$

$\dot{U}_{D}{ }^{\circ} \dot{U}_{1}=\dot{U}_{2} \dot{U}_{E}{ }^{-} \dot{U}_{D}{ }{ }^{-} \dot{U}_{D}{ }^{-} \dot{U}_{C}$

then $\dot{U}_{E}=2 \dot{U}_{D}{ }^{-} \dot{U}_{C}=2 \dot{U}_{2}{ }^{-2} \dot{U}_{1}$

So $\dot{I}_{2}^{\prime}=\frac{\dot{U}_{F}{ }^{-} \dot{U}_{E}}{R}=\frac{\dot{U}_{2}{ }^{-2} \dot{U}_{2}{ }^{2} \dot{U}_{1}}{R}=\frac{{ }^{2} \dot{U}_{1}}{R}-\frac{\dot{U}_{2}}{R}$

Thus the part of the circuit parameters for $\mathrm{Y}_{1}$ is:

$\left[Y_{1}\right]=\left\{\begin{array}{cc}-\frac{1}{R} & 0 \\ \frac{2}{R} & -\frac{1}{R}\end{array}\right\}$

In figure 3 there are:

$\dot{I}_{1}=-\dot{I}_{2}$

$\dot{\boldsymbol{U}}_{1}=\dot{\boldsymbol{U}}_{2}+R \stackrel{\boldsymbol{I}}{\mathbf{I}}_{1}^{\prime \prime} \quad \stackrel{\boldsymbol{I}}{2}^{\prime \prime}=-\frac{1}{R} \dot{\boldsymbol{U}}_{1}-\frac{1}{R} \dot{\boldsymbol{U}}_{2}$

So parameters $\mathrm{Y}_{2}$ is:

$[Y]=\left[Y_{2}\right]+\left[Y_{1}\right]=\left\{\begin{array}{cc}0 & -\frac{1}{R} \\ \frac{1}{R} & 0\end{array}\right\}$

Network parameters for general Y: 


$$
\left[Y_{2}\right]=\left\{\begin{array}{cc}
\frac{1}{R} & -\frac{1}{R} \\
-\frac{1}{R} & \frac{1}{R}
\end{array}\right\}
$$

Formula (4) of the parameters, it is shown in the Y rotation. Therefore the circuit diagram shown an editor. Its rotation coefficient is $\frac{1}{R}$. Generally, due to the influence of resistance components, with two error amplifier and partial resistance level to realize incurvate elements can have certain difficulty. According to the theoretical analysis and experimental results prove that the actual realization circuit should turn the correction, as figure 4 shows.

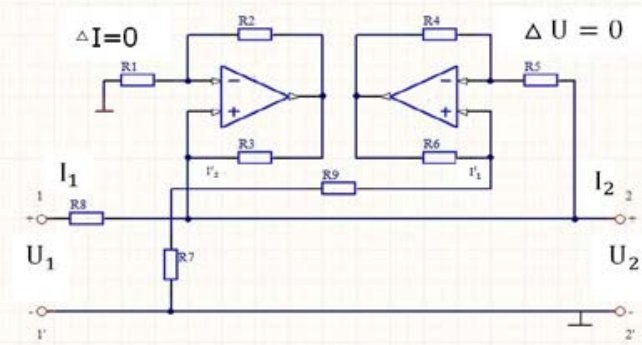

Figure 4: Correction Circuit of Gyrator

\section{Study on memory resistors}

So far, the general electric, electronics and related disciplines in every field, although constitute the basic model, phyletic and various. But the basic components comprise circuit, either resistors R, inductors $\mathrm{L}$ and capacitor $\mathrm{C}$ three.

The three basic elements from the study of the relationship between constraints, will have a very important inspiration, thus the existence of resistors are memory.

1. Resistors. In 1826 German physicist named ohms proved that:

$\mathrm{u}(\mathrm{t})=\mathrm{R}_{\mathrm{i}}(\mathrm{t})$

In u-i plane, the relationship of a line for through the origin, this is the ohm's law. If the line of slope do not change over time, it is called constantly resistance.

If this concept is developed and deepened, the element in any time t, the voltage and current relationship by u-i can be a curve to determine the plane, the component is called resistors, the constraint equations is:

$\mathrm{f}(\mathrm{u}, \mathrm{I}, \mathrm{t})=0$

So, if the resistance is instantaneous components or no memory components. The current i product voltage $\mathrm{u}$, power is expressed as:

$Q=u_{i}$

Inductor. In 1831 British physicist Faraday proposed that inductors is inductance coil model. It is the magnetic flux chain $\psi$ and current $\mathrm{i}$ in the relationship, $\psi-i$ used a plane curve to determine. The curve of the slope is inductive components. The constraint relation is:

$f(\psi, i, t)=0$ or $u(t)=\frac{d \psi}{d t}=L \frac{d i}{d t}$

So we can know, The formula shows that in any time t, inductive components on magnetic flux chain $\psi(t)$ is relative with the voltage $u(t)$ from the moment $-\infty$ to $t$, it shows that $\psi(t)$ has the function of memory.

In reference voltage and current under the direction of the elements, at any time the power of this component is:

$$
P=u_{i}-L_{i} \frac{d i}{d t}
$$

In a moment the stored energy in inductive components is: 


$$
W L(t)=\int_{-\infty}^{t} u i d \tau=\int_{-\infty}^{t} L i \frac{d i}{d t} d \tau=\frac{1}{2} L i_{i}^{2}=\frac{1}{2 L} \psi^{2}(t)
$$

Capacitors. In 1821 French mathematicians Poisson proved the energy storage on the capacitor. The model of capacitor is capacitors. Its charge $q(t)$ is related with voltage $u(t)$, in $q-u$ plane it can be determined by a curve. The curve of the slope is capacitance components. The constraint equations are:

$$
f(q, u, t)=0 \text { or } i(t)=\frac{d q}{d t}=c \frac{d u}{d t}
$$

This shows, at any time t, capacitance components charge $q(t)$ is relate with the current $\mathrm{i}$ from the moment $-\infty$ to $\mathrm{t}, \mathrm{q}(\mathrm{t})$ has the function of memory.

In the current and voltage reference direction, at any moment power of components is:

$$
P=u_{i}-c u \frac{d u}{d t}
$$

In a moment stored energy of Capacitance components is:

$$
\mathrm{Wc}(\mathrm{t})={ }_{W C}(t) \int_{-\infty}^{t} u i d \tau=c \int_{-\infty}^{t} u d u=\frac{1}{2} c u^{2}(t)=\frac{1}{2 c} q^{2}(t)
$$

The basic variables which constitute the circuit are voltage $u(t)$, current $\mathrm{i}(\mathrm{t})$, charge $q(t)$ and magnetic flux chain $\psi(t)$. In the four variables, $u(t)$ and $\psi(t), i(t)$ and $u(t)$ respectively follow the below rules:

$$
\left.\begin{array}{l}
u(t)=\frac{d \psi(t)}{d t} \\
i(t)=\frac{d q(t)}{d t}
\end{array}\right\}
$$

The formula shows that $u(t)$ and $\psi(t), i(t)$ and $q(t)$ are not independent, in other words, $u(t)$ and $\psi(t)$, $i(t)$ and $q(t)$ are variables each.

If the above four variables of the amount for every two to constitute variables, the four variables have six possible group form, as shown in figure 5.

In the six possible combinations, due to the variable coupling $(u, \psi)$, and $(i, q)$, so only $(u, i),(I, \psi)$, $(u, q)$ and $(\psi, q)$ are not dynamic. The four variables of implicit in the first three respectively represent the three basic elements of known, namely resistance $R$, inductance $L$ and capacitance $C$. As shown in figure 5, characteristics of the corresponding components is shown by the (1), (2), (3) three lines. As the same reasons, according to completely similar physical line (4) should also be representative of the determination of components. Although it is not same with the three basic elements, its characteristic can be expressed by a curve in $\psi-q$ plane.

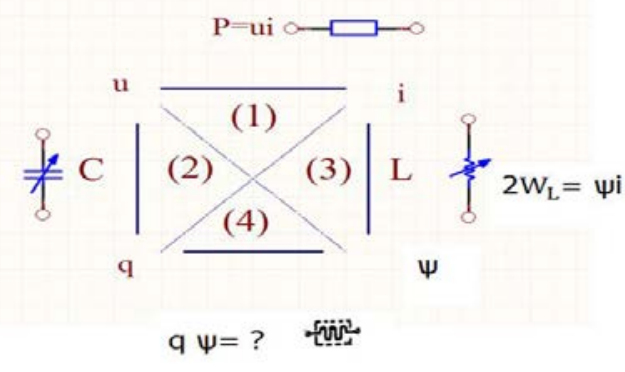

Figure 5: Three Novel Types of Basic Components

Obviously, in the basic circuit components, in addition to R, L, C, There is kind of another component which also describe the relationship of $\psi-q$ element, we called this kind of another component Memritor resistor.

The exists of the memritor resistor is logical reasoning, science respecting and reasonable prove. This is same that after the Great Russian chemistry Dmitri Ivanovich Mendeleev invented the chemical element, he also inferred that there are some chemical elements which not been discovered.

Memritor resistor. If Memritor resistor is looked as the two end components, and expressed with a curve in a plane $\psi-q$ : 
$f(\psi, q, t)=0$

With the characteristic of $\psi$ and q, Memritor resistor has the capacity of memory.

$$
\begin{gathered}
\psi(t)=\int_{-\infty}^{t} u(\tau) d \tau \\
q(t)=\int_{-\infty}^{t} i(\tau) d \tau
\end{gathered}
$$

As the above two formula shown, the value of $\psi(t)$ and $q(t)$ is related with $u(t)$ or $i(t)$ from the moment of actuators $-\infty$ to $\mathrm{t} . \psi$ and q respectively have the function of memorize the voltage and current, and its memory time limits for infinite.

If Memritor resistor curve is expressed to characteristics of charge control in $\psi-q$ plane, then we have $\psi=f(q)$. According to the formula 5 :

$u(t)=\frac{d \psi}{d t}=\frac{d \psi}{d t} \bullet \frac{d q}{d t}=M(q) \bullet i(t)$

In formula 6, $M(q)=\frac{d \psi}{d q}$ is named incremental memristancc

Formula 6 shows the relationship between the u and i which can be seem as generalized ohm's law, and the value of $M(q)$ at any moment depends on the total charge. Similarly, if the characteristic of Memritor resistor is expressed to $q=h(\psi)$, which named chain control characteristics. By formula 5:

$i(t)=\frac{d q}{d t}=\frac{d q}{d \psi} \cdot \frac{d \psi}{d t}=W(\psi) \bullet u(t)$

In formula $7, w(\psi)=\frac{d q}{d \psi}$ ) is named incremental memductancc.

In generalized dynamic system, the characteristic of Memritor resistor can be expressed to:

$$
\left.\begin{array}{c}
\dot{X} \\
Y=f(x, u, t) \\
Y=q(x, u, t)
\end{array}\right\}
$$

Formula 8 can be analyzed by inductance resistance.

\section{Conclusion}

In summary, as a new-type element, Memritor resistor is being understood and utilized.

\section{References}

[1] LI Jun, JIA Xinzhang. Creation of a Sub-Circuit Model for PSpice[J].Microelectronics. 2004, 34(3)12-19.

[2] WU Shaoqin. Modeling for electronic parts in circuit simulation[J]. Electronic Design Engineering, 2009,17(8)88-91.

[3] SHENG Dingyi,Yang Geng,et al. A New Power PIN Diode PSpice Subcircuit Model[J]. MODERN ELECTRONICS TECHNIQUE, 2008, 31(6)108-109.

[4] LIU Yan, ZHANG Qilin et al. The Full Current Model of MOV Modeling with PSPICE[J] Science Technology and Engineering, 2014, 14(2)112-119. 